MATEC Web of Conferences 22,05024 (2015)

DOI: $10.1051 /$ matec conf/ 20152205024

(C) Owned by the authors, published by EDP Sciences, 2015

\title{
Research on Torque Loading Technology Based on L Lever in Space- craft Bolt
}

\author{
Xiaohui Song, Zhibin Liu \& Zhe Liu \\ Beijing Institute of Spacecraft Environment Engineering, Beijing, China
}

\begin{abstract}
As the main form of structure assembly, the stability of bolt joint is directly related to the performance of spacecraft. This essay is used to analyze the cause of standard torque spanner which is not suitable to the tightening torque and summarized according to different operating conditions. Based on thorough analysis of fastening principles and principle of type serialization, a scheme of integrated L Lever is established. The results of experimentation indicate that the scheme of integrated L Lever is reasonable along with the requirement of developing long-life and high-reliability spacecraft. Therefore, the stability of fasteners is greatly enhanced.
\end{abstract}

Keywords: L Lever; spacecraft; bolt; torque

\section{INTRODUCTION}

All forms of threaded joint are massively applied in the area of spaceflight, especially during general assembling stage of spacecraft. Threaded joint can affect the performance of spacecraft directly even decide the success or failure of one spacecraft mission. Therefore, it has become the focus of spacecraft development process. Generally speaking, exerting preload to the threaded fastener is the common method to guarantee the high stability of threaded joint as well as to meet the requirement of mechanical environment during development. Usually, the torque control is adopted to control the preload of threaded fastener, that is to say, the torque tightening device is used through the changing tightening torque values $(T)$ to control preload of threaded joint $(F)^{[1]}$.

As stated above, the torque tightening device is important during spacecraft assembly, it is an essential online testing device and assembly equipment during developing high-quality and long-life spacecraft. It also plays a key role in guaranteeing the assembling quality of spacecraft. The most commonly-used torque tightening device during spacecraft development is the preset torque wrench. GEDORE DREMOMETER 753 and GEDORE DREMOMETER 8560 are most common in active use.

The preset torque wrench plays a positive role during spacecraft development as well as meets the loading requirement of preloading force of fastener at most operating conditions. However, situations such as the torque tightening that cannot be exerted to fastener have increased due to limited space with increasingly complicated spacecraft structure. Breakdowns such as screw-off or breakage could happen if the preloading force of fastener did not meet the requirement of design ${ }^{[2]}$

This essay analyzed the problem that the torque loading cannot be exerted to spacecraft fasteners and found out a solution based on L Lever which solves the problem efficiently.

\section{THREADED FASTENING PRINCIPLE}

During installation of fasteners, the tightening force of screw is transformed into preloading force compressing the screw thread form axial direction through the relative movement of screw thread pair. Reliability of screw joint mainly depends on the preloading force between fasteners and base whose major form is tensile force. Generally speaking, when fasteners, the connected members and base are stable, the greater the tightening force exerted to screw, the greater preloading force is; the tighter the two connected screws are, and the more stable of the screw joint is. The relationship between $\mathrm{T}$ and $\mathrm{F}$ can be expressed as follows: $T=K d F$

In this formula, $K$ means the tightening torque coefficient and $d$ means the thread nominal diameter.

When using the same standard screw, the tightening torque of screw is affected by two factors: One is the reasonable preloading force and the other is the tightening coefficient.

The theoretical formula of tightening torque coefficient $\mathrm{K}$ can be expressed as follows ${ }^{[3]}$ :

$$
\begin{gathered}
K=\frac{1}{2}\left[\frac{d_{2}}{d} \tan (\varphi+\rho)+\frac{2 \mu_{n}\left(D_{1}^{3}-D_{0}^{3}\right)}{3 d\left(D_{1}^{2}-D_{0}^{2}\right)}\right] \\
\varphi \text { means the lead angle, } \varphi=\arctan \left(p / \pi d_{2}\right), \rho
\end{gathered}
$$

means the vice equivalent frictional angle of threaded connection, generally speaking, $d_{2}$ means the pitch diameter of thread, $\mu_{n}$ means the combination coef- 
ficient of friction between screw and the fitting surface of connected one.

$r_{n}$ means the torus equivalent friction radi$\operatorname{us}\left(r_{n}=\left(D_{1}^{3}-D_{0}^{3}\right) /\left[3\left(D_{1}^{2}-D_{0}^{2}\right)\right]\right)$ in this formula.

For the specification of fasteners which is fixed during the general design process of spacecraft, therefore, the nominal diameter of screw $(d)$ is of a certain value. The tightening coefficient of screw is mainly affected by the friction between screw and thread pair of the connected one and the friction between screw head and the surface of the connected one.

Generally speaking, we use the hexagonal titanium screws as standard screw on spacecraft and the aluminum plate skin cellular structure as matrix and the aluminum alloy plate as the connected material. When screw, matrix and the connected material are stable, the tightening coefficient $(\mathrm{K})$ is a certain value. A typical connecting mode on spacecraft is shown in Figure 1 and Figure 2 showed the pull-up curve during the installation of screw. The standard of fasteners is M3, and we use the ML30CrMnSiA material of $880 \mathrm{MPa}$ strength grade, the $1 \mathrm{Cr} 18 \mathrm{Ni}$ Ti stainless steel as matrix and the $5 \mathrm{~A} 06$ aluminum as connected material ${ }^{[4]}$.

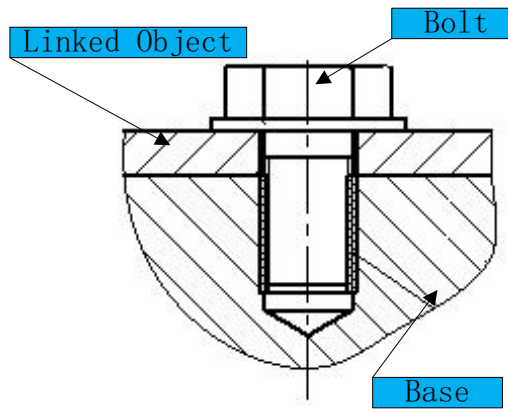

Figure 1. Pull-up curve of screw installation

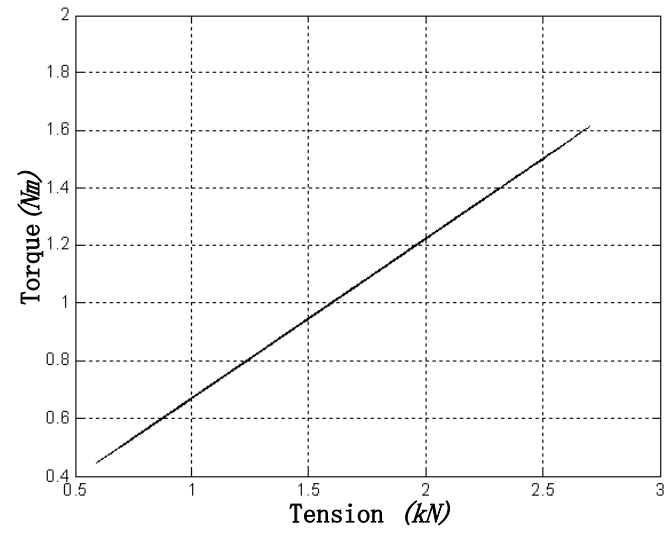

Figure 2. Pull-up curve of screw installation

It is easy to tell that the stretching resistance is basically liner with preset torque, that is to say, it is possible to control the preloading force of fasteners as long as fasteners are installed reasonably. Ensure a firm installation of fasteners and stability of connected material.

\section{CURRENT PROBLEMS}

The Torque loading which exerts to fasteners is the main method of controlling tightness of screw during spacecraft manufacturing process. Since the structure of spacecraft is becoming more and more complicated, tools to conduct torque tightening cannot operate normally due to a limited space and lead to the situation that standard tools are too big for available space. This essay reduces the key problem to two kinds of situations through deeply analysis of this kind of problem. One is caused by the limited axial space of fasteners, in another words, the spare effective height of axis direction is less than the sum of torque spanner and the height of adapter. And another reason is the limited radial space of fasteners, that is to say, the spare space of diameter direction is less than the swing diameter of the head of torque spanner.

\section{SOLUTION}

Both of those two situations can come down to oversize of torque spanner and rotary pole for it cannot move inside the spare space of target fastener. Under the precondition that the operation space remains unchanged, decreasing the size of the head of torque spanner to adapt to the requirement of operation space is the only solution.

Under the condition of limited axial space, the vertical connecting rod that connected the head of spanner and screw together should be as short as possible, so that it can fit into the limited axial operation space.

As for limited radial space, the head of spanner should be reduced through radial direction or retreat it outside the effective operation space.

The torque spanner which is usually used at present takes too much operation space. Take GEDORE DREMOMETER 753 as example, its head altitude is about $50 \mathrm{~mm}$ and its radius is about $15 \mathrm{~mm}$. According to research which aims at spacecraft fasteners, the required minimum axial space is about $16 \mathrm{~mm}$ and the required minimum radial space is about $10 \mathrm{~mm}$, that is to say, current existing torque spanners cannot meet that requirement.

After deeply research, the author finds out that the structure of GEDORE4400 torque spanner is different from normal torque spanners. Its moment output part can be moved away from the connecting part with fasteners and combined with L-shaped connecting rod so that it is possible to work in limited space. 
One-piece L-shaped connecting rod is rigid and has adjustable length so it can meet the requirement of limited space. Transition fit which is adopted in this kind of torque spanner is to minimize system error due to cooperation. Figure 3 is the sketch map of the combination of one-piece L-shaped connecting rod joint with the socket head cap screw.

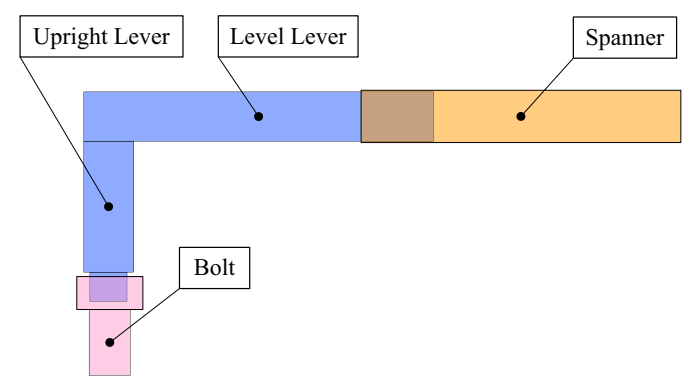

Figure 3. Sketch map of the combination of one-piece L-shaped connecting rod joint with socket head cap screw.

\subsection{Design of L-shaped rod}

To some points, the torque wrench through tooling is a tool used with connecting screw and torque spanner. Its main purpose is to acquire effective connection and operation space through transfer, so it can control tightening torque of fasteners effectively.

According to design philosophy of serialization, modularization and parameterization, the transfer that connected to GEDORE4400 torque spanner is composed by horizon bar and vertical bar. The integrated design of it can decrease errors during connecting. In this device, the vertical bar is used to be connected with target fasteners and the horizon bar is used to be connected with the torque spanner.

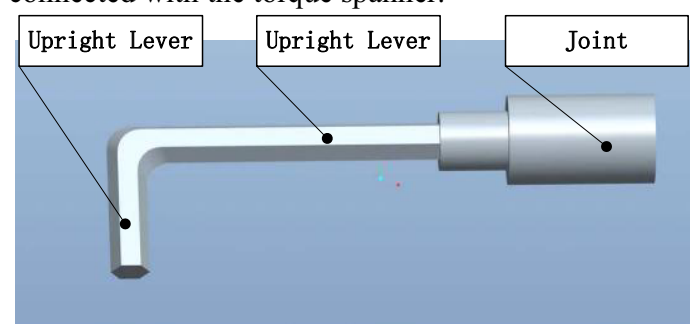

Figure 4. Sketch of one-piece L-shaped transfer

Figure 4 is the sketch of one-piece L-shaped transfer. Assume that the effective length of vertical bar is L1 and the effective length of horizon bar is L2, the adapter sleeve is designed to a cylinder of $\Phi 15 \mathrm{~mm}$ according to the shape of torque spanner. And then the locked horizon bar and the adapter sleeve go through screw at the same time. For the cross-section shape of $\mathrm{L}$ Lever is a regular hexagon, it can match with the socket head cap screw perfectly. The author developed a set of transfer through different combinations of L1, L2 and different sizes of cross-section to meet the need of different condition.

During spacecraft development, a L-shaped cross-section shape can fit into the hexagon socket cap screws of M3, M4, M5, M6 and M8 and has four kinds of length of vertical bar including $15 \mathrm{~mm}, 20 \mathrm{~mm}$, $30 \mathrm{~mm}$ and $50 \mathrm{~mm}$; it also has four kinds of length of horizon bar, including $50 \mathrm{~mm}, 80 \mathrm{~mm}, 100 \mathrm{~mm}$ and $120 \mathrm{~mm}$.

Figure 5 is a model of one-piece L-shaped transfer.

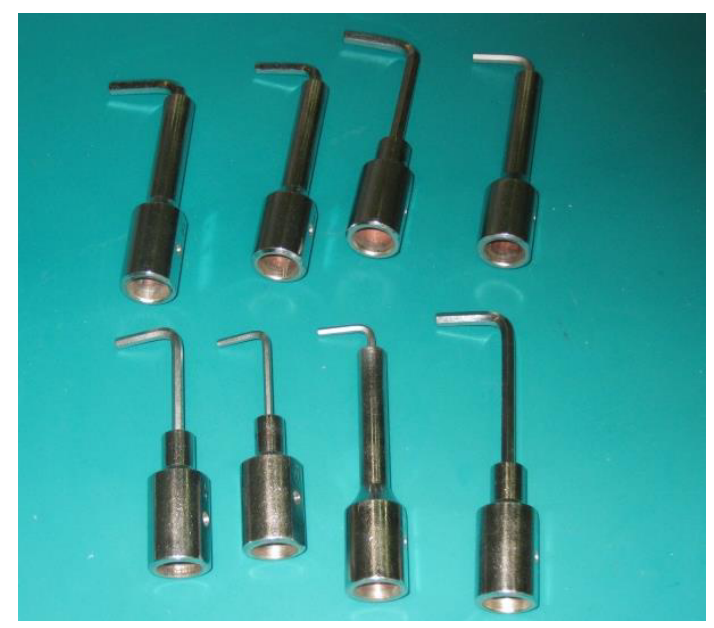

Figure 5. One-piece L-shaped lever

\subsection{Finite element analysis}

L-shaped transfer is built with T9 chisel tool steel through one-time forming. The author used ANSYS software to run a mechanical simulation test of $\mathrm{L}$ lever and find out that when the tightening torque of M4 screw reaches its maximum $2.5 \mathrm{Nm}$, the stress distribution should be as what is shown in Figure 6. That is to say, the maximum stress should be $870 \mathrm{~Pa}$ and occur at the joint part of vertical bar and horizon bar. Based on this condition, the intensity meets the requirement of torque load and no damage would occur. 


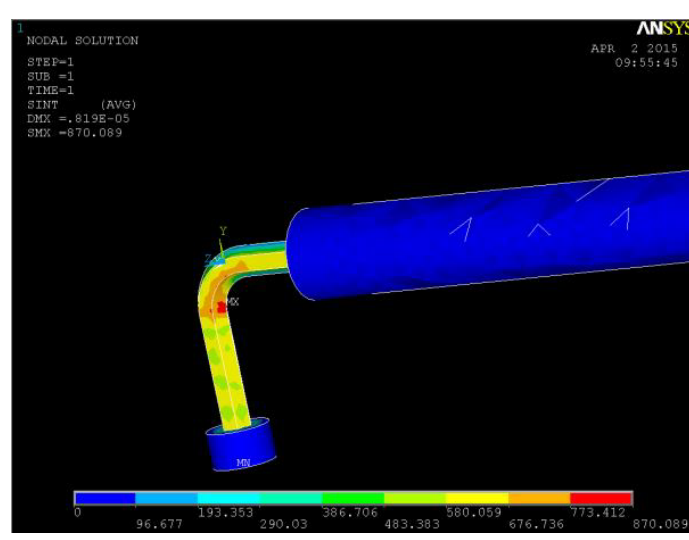

Figure 6. Stress diagram of $\mathrm{L}$ lever

\section{EXPERIMENTAL VERIFICATION}

The length of arm changed after combination of one-piece L lever and torque spanner which may result in the difference between theoretical torque transfer coefficient and practical torque transfer. So the author designed a calibration test for tool combination of torque load and operated according to standard moment method. Through this test, the author obtained the data and the transfer coefficient through analyzing the relationship between output torque of torque spanner and the transfer, input value of torque spanner and the length of transfer.

\subsection{Test scheme}

The equipment used in this test is 8624-001 torque tester which has a test precision of $0.001 \mathrm{Nm}$, the torque spanner and the one-piece transfer are regarded as test objects. Figure 7 is the effect picture of test for torque spanner and one-piece transfer. During the test, the operator hold torque spanner in clamps way to minimize personal error and random error and keep vertical bar perpendicular to torque marking apparatus transfer and increase strength slowly and steadily.

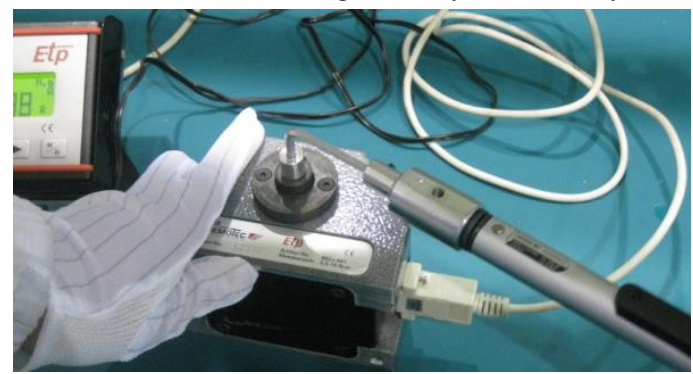

Figure 7. Effect picture of test for torque spanner and one-piece transfer

\subsection{Data analysis}

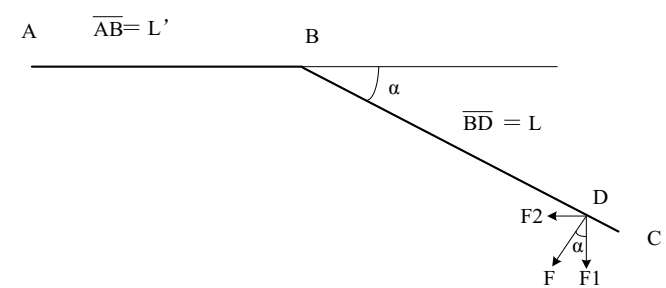

Figure 8. Stress analysis diagram of torque spanner plus one-piece L lever

Figure 8 is the sketch map of connected relationship of torque spanner and L-shaped transfer. Line segment AB stands for horizon bar and its effective length is $L$ '; line segment BC stands for the force arm of torque spanner; point $\mathrm{D}$ stands for the point of force application when operator uses torque; line segment $\mathrm{BD}$ stands for the distance between the point of force application to the center line of tenon and its effective length is $L ; \alpha$ stands for the included angle between horizon bar and hand shank of torque spanner and $F$ stands for the stress that exerted form point $\mathrm{D}$ which is vertical to line segment $\mathrm{BC}$.

The relationship between the moment $M^{\prime}$ that the external force $F$ exerted to point $\mathrm{A}$ and the moment $M$ that exerted to point $\mathrm{B}$ is deduced from the precondition that the torque spanner plus transfer system is in statics balance ${ }^{[5]}$.

$$
\begin{array}{rcc}
M^{\prime} & = & F \sin \alpha L \sin \alpha+F \cos \alpha\left(L^{\prime}+L \cos \alpha\right) \\
& = & F \sin ^{2} \alpha L+F \cos \alpha L^{\prime}+F \cos ^{2} \alpha L \\
= & F L+F L^{\prime} \cos \alpha \\
= & F\left(L+L^{\prime} \cos \alpha\right)
\end{array}
$$

$M=F L$

$$
\begin{aligned}
& K=\frac{M^{\prime}}{M}=\frac{F\left(L+L^{\prime} \cos \alpha\right)}{F L}=\frac{L+L^{\prime} \cos \alpha}{L} \\
& M^{\prime}=K M=\frac{L+L^{\prime} \cos \alpha}{L} M=\left(1+\frac{L^{\prime} \cos \alpha}{L}\right) M
\end{aligned}
$$

We may get to know the relationship between the out value of torque spanner $M^{\prime}$ and the torque moment input value $M$ depends on the length of torque transfer horizon bar $L^{\prime}$, the length of the force application arm of torque spanner $L$ and the included angle between horizon bar and the application arm of torque spanner $\alpha$. Measure the effective length of transfer horizon bar and torque spanner, then, calculate theoretical transfer coefficient $\mathrm{K}$ according to the formula as follows: 


$$
K=\frac{L+L^{\prime} \cos \alpha}{L} .
$$

By comparing the data from theoretical calculation and experiment, we may find out that there are some errors between theoretical transfer coefficient and practical transfer coefficient. The main reason is that at least one transfer link is increased after using the transfer system. Besides, friction caused by coordinate between the torque spanner and the transfer tool can lead to certain system error and some uncertain angle errors between the horizon bar and the torque spanner may bring errors to transfer coefficient system.

Through a set of calibration test for torque spanner and corresponding transfer tool, we may find out that the measured value of output torque of $L$ lever is of $88 \%-98 \%$ of the theoretical value. Take system errors into consideration, we may come to the conclusion that the torque transfer coefficient of L lever tool is at a reasonable scope.

\subsection{Discussion of test result}

Through a set of calibration test for torque spanner and corresponding transfer tool, we may find out that the measured value of output torque of $L$ lever is of $88 \%-98 \%$ of the theoretical value. Take system errors into consideration, we may come to the conclusion that the torque transfer coefficient of L lever tool is at a reasonable scope.

To ensure the veracity of $K$, following measures should be taken:

1) Try to minimize the ratio of the effective length of horizon $L$ ' and the arm length of torque spanner $L$, that is to say, give priority to combination of one-piece $\mathrm{L}$ transfer plus torque spanner with smaller ratio between $L^{\prime}$ and $L$.

2) Try to keep the torque spanner in line with the horizon bar $(\alpha=0)$ so errors due to measurement would have less impact to $K$. Based on this condition, the formula of transfer coefficient can be simplified as follows:

$$
K=\frac{L+L^{\prime} \cos \alpha}{L} \text {. }
$$

\section{APPLICATION TO SPACECRAFT}

For the problem caused by limited space of axial direction, we may choose different height of vertical bar of one-piece L lever according to the spare space of axial direction of fasteners, and then connect it to a certain length of horizon bar. According to the research of different model of fasteners, the author finds out that the minimum space of its axial space is $16 \mathrm{~mm}$, so we may set the minimum length of vertical bar to $15 \mathrm{~mm}$ in order to fit in.

This kind of one-piece L lever used the same regular hexagon as fasteners so it won't cause problem such as the limited radial space.
The installation progress is shown as follows:

1) Ensure enough space: It means the effect spare space could affect the installation of fasteners. It is also used as criterion when choosing the size of torque transfer tool.

2) Choose torque loading tool: It means to choose a suitable one-piece $\mathrm{L}$ transfer tool according to the need of space.

3) Choose torque transfer tool: It means to choose a suitable standard one-piece $\mathrm{L}$ transfer tool according to the space.

4) Set the indicating value of torque: It means to calculate the moment value of torque according to the transfer coefficient of target fasteners and selected transfer tools.

5) Torque loading: It means to use previous required torque loading tools to tighten the fasteners after adjust the indicated value of torque.

\section{CONCLUSION}

This essay designed a one-piece $\mathrm{L}$ lever that aimed at the problem of standard torque spanner which is not suitable to the tightening torque during the spacecraft development and the principle of serialization. It featured strong university and flexibility. This essay also verified the application effect through theoretical calculation and experiment. The set of device can meet the requirement of long-period and high reliability of spacecraft development; it also increases the reliability of fasteners installation effectively.

\section{REFERENCES}

[1] Honggang Lei. 2001. The Expert Treatment System of Fatigue Loading Spectrum for Large-Span Grid Structures. Proceedings of Sixth Pacific Structural Conference.

[2] Tajinma, J. 1964. Effect of Relaxation and Creep on the Slip Load of the High-Strength Bolted Joints, Structural Design Office, Japanese National Railways, Toyko, June.

[3] Denkhaus, H.G. 1961. Strength of washers, Council for Scientific and Industrial Research contract. No. CN378, The National Mechanical Engineering Research Institute, Council for Scientific and Industrial Research, Pretoria, South Africa, Feb..

[4] Xu Fuxiang. 2002. Satellite Engineering, China Aerospace Press.

[5] Robert D T. \& Michael E. 1994. Nonlinear finite elment evaluation of marman clamp structural capacity. AIAA 294213462 CP: 320-330. 